\title{
Dance movement therapy in Asia: an overview of the profession and its practice
}

\section{亚洲舞蹈治疗回顾与总览}

\author{
Tony Yu Zhou ${ }^{1}$, Nayung Kim², Shoichi Machida ${ }^{3}$, Yukari \\ Sakiyama ${ }^{3}$, Pei-Shan Tsai ${ }^{4}$, Tsungchin Lee ${ }^{4}$, Rainbow \\ Tin Hung $\mathrm{Ho}^{5}$, Rashi Bijlani ${ }^{6}$, Devika Mehta ${ }^{6}$, Minh Bui ${ }^{7}$ \\ ${ }^{1}$ Inspirees Institute, China, ${ }^{2}$ South Korea, ${ }^{3} \mathrm{~J}$ apan, ${ }^{4}$ Taiwan, \\ ${ }^{5}$ Hong Kong, ${ }^{6}$ India, ${ }^{7}$ Vietnam
}

\begin{abstract}
This article, co-written by several dance movement therapy professionals in Asia, gives an overview of the history, training, professional association, professional practice, research, strengths and challenges of dance movement therapy in Asia - particularly in East Asia and Southeast Asia. Some cultural reflections are included to offer the Asian perspectives.
\end{abstract}

Keywords: Dance movement therapy, DMT, Asia, training, professional association, professional practice, research, psychotherapy

\section{摘要}

本文通过亚洲的数位舞蹈治疗专业同行概述和总结了亚洲，特别是东亚和东南亚舞蹈动 作治疗的历史，专业培训，专业协会，专业实践，科研，以及这一地区面临的优势及挑 战。文章还特别加入了一些文化面向上的思考和方式，以反映来自亚洲的视角。

关键词: 舞蹈治疗，亚洲,培训，专业组织，专业实践，科研，心理治疗

\section{Introduction}

"West Meets East" is an everlasting subject of inspiring discussion throughout human history (Northrop, 1946; Needham, 1964; Russell, 1922; Wells, 1971). In the past 5,000 years, West and East have been playing the leading role alternately in the world (Needham, 1964). Although the discipline of DMT (when placed within the context of human history) has existed for only a very short period of time, dance and movement - as the basic vehicle and form of self-expression - has always been present in everyday life.

The Asia defined here primarily covers East and South Asia (China/Taiwan, India, Japan, South Korea and Vietnam). The primary reason for grouping these countries and regions together in such a way is because they share some culture and historical roots based on Confucian, Taoism and/or Buddhism. From ancient times, Chinese, Japanese, Indian, South Korean and Vietnamese cultures have considered both the body and mind 
to be a unity and not separated. In its wider definition, the 'body' includes both physical and spiritual aspects. All four family-centred cultures emphasized both a sense of collectiveness and a relationship with other environments - e.g. people and nature. The body itself exists within in that space where we have relations with others. Some good body/mind practices like Yoga, Tai'chi and meditation etc. were originally developed in Asia and are recognised, now, as having contributed substantially to the present somatic field.

\section{China mainland/Hong Kong/Taiwan}

The roots of DMT lie in the integration of body, mind and spirit- aspects of the self reflected in Taoism, Confucianism and Buddhism (defined as Three Teachings) and the backbone of Chinese philosophy and culture (Wells, 1971).

The thorough study of Three Teachings is a vital preliminary step to establishing any real intellectual and moral community between the great people of the Eastern and Western worlds. The Three Teachings are complementary to each other and satisfy the somatic, intellectual and emotional needs of the Chinese people. For example, Taoism one of the oldest philosophies in ancient China - focuses on nature and the somatic experience in the present. The social body - comprising the community, the village and the land - corresponds in all aspects to the physical body in Taoism. In both, the survival of humanity is decided in the "here and now." According to Schipper (1994), "My destiny is within me, not in Heaven!" Confucianism is a practical dogma of ethical behavior defining the order of human society. It focuses on the mind without religious doctrine and persecution since extremes and aggressiveness are not appreciated in Confucianism. Interestingly, Taoism and Confucianism - the two indigenous 'religions'-are not exclusive but compatible and created a greater acceptance of Buddhism which came from India. Buddhism, with its increased focus on spirituality, became a necessary spiritual power for the Chinese people and helped them to deal with challenges (e.g. facing death in the Chinese culture). The essence of DMT - body, mind and spirit - are intrinsically linked to Taoism, Confucianism, and Buddhism respectively. It is therefore not surprising that DMT has easily gained a natural and strong resonance among Chinese people.

China is eager to acquire Western learning - not simply in order to acquire

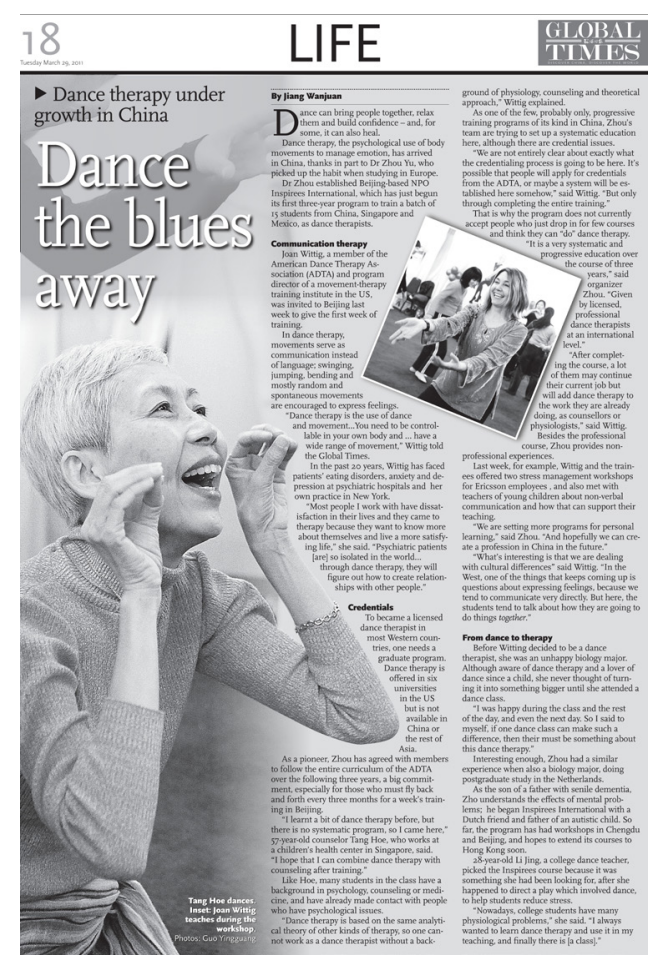


national strength but because a very large number of people consider learning a good thing in itself. In China, it is traditional to place high value on the acquisition of knowledge. Many students travel to the West to study; when they return to China, they bring back Western habits and perspectives and so are responsible for rapidly modernizing the Chinese outlook.

Such modernization has proved important for, in earlier decades, China's traditional civilization had gradually stultified - ceasing to produce much of value in the way of art and literature. This was not due to any decadence in the race, but merely to a lack of new material. The gradual influx of Western knowledge, however, began to provide just the stimulus that was needed (Russell, 1922). This stimulus can be considered as the catalyst rather than the source of social development and the advancement of Chinese society. Dance/movement therapy has awakened the consciousness of the Chinese people enhancing their awareness of the value of their own culture and wisdom; more importantly, it connects the East and the West in an equal way.

The first Western-oriented dance therapy was introduced to mainland China by Yulan Fucius, BC-DMT, who returned to Beijing in 1995 to begin her practice. Her aim was to combine dance therapy with traditional Chinese medicine teachings. In 2004, Dr. Tony Yu Zhou brought Dutch dance therapist Zvika Frank to China to begin pioneering work in dance therapy within the country. Thanks to these pioneers, dance therapy has gained an increasingly important profile in China. By 2011, two professional dance therapy training programs had been established in China with the aim of training local dance therapists. Dance therapy began to gain momentum and developed quickly in China - in synchrony with growing international developments. In Taiwan, the origins and development of dance therapy were traced back to 1982 when Lee Tsungchin was invited to work as a dance therapist at the psychiatric department of Taipei Veterans General Hospital (Lee, 2014). Since then, the clinical application of dance therapy in Taiwan has continue to flourish. After the 921 Earthquake (also known as the Jiji Earthquake), Professor Lee was invited by the Performance Art Alliance to take on the role of convener of the Art and Psychology Group. Together with Professor En-Chang $\mathrm{Wu}$ and Professor Heng Ping, the Convener of the Psychology Group and the Art group respectively, the importance and effect of the arts as therapy was directly and indirectly introduced to many who could benefit from it. In Hong Kong, the leading figure in dance therapy is Professor Rainbow Ho who has been trained in the US and has obtained her BC-DMT credential and CMA title. She has been pioneering dance therapy and developing her work since 2002 in The University of Hong Kong.

China initiated two professional dance therapy certification training programs in 2011 - one ADTA Alternate Route (Inspirees Institute) and one German Dance Therapy Association Program (Apollo). Both are non-academic professional development programs out of university but have been developed in compliance with guidelines established by international professional associations. The ADTA program is a postgraduate program and thus requires studying psychology courses at graduate level as well as completing coursework in dance therapy and undertaking $700 \mathrm{hrs}$ of internship in clinical settings. So far, more than 80 students have been fully trained in the two programs - with four students gaining dance therapy credentials abroad. Some Chinese 
universities are expressing a growing interest in dance therapy but so far no academic program has been set up. However, several universities have now begun running dance therapy courses for both undergraduate and graduate students, in order to prepare themselves for starting a dance therapy program in the future. In Taiwan, Professor Lee Tsungchin developed dance therapy courses in National Chengchi University and a certified training program out of the university in conjunction with the Taiwan Dance Therapy Association. No academic dance therapy training program exists yet in Hong Kong. Dance therapy courses are available, however, at the Centre on Behavioral Health and also under the Master of Social Sciences (Behavioral Health) and the Master of Expressive Arts Therapy at the University of Hong Kong.

Due to strict regulations enforced by the government, China has not yet established an official professional association. By law, only the association registered with Ministry of Civil Affairs in mainland China is legally recognized. In 2016, the Chinese Arts Therapy Group (CATG) was established and led by Dr. Tony Yu Zhou, under the auspices of the Chinese Psychological Association. A working division on dance therapy has been set up to focus on the registration and standardization (including the development of a code of ethics) of dance therapy in China. This group is yet to grow and integrate with other organizations in China. In 2002, the Taiwan Dance Therapy Association was established in response to the growing need for a professional forum for dance therapy. Since 2008, professional certifications of training in dance therapy have been issued by the Academic Committee of Taiwan Dance Therapy Association under a formal review procedure. There are currently 25 certified dance therapists - including those trained in Taiwan and those trained in both the UK and the USA. The Hong Kong Dance Movement Therapy Association was established in 2014.

DMT is not strictly regulated in China as it is a new profession and, as such, is not yet fully recognized. By law, however, psychotherapy has to be conducted in hospitals under the supervision of medical professionals; this applies to both Chinese dance therapists and foreign dance therapists working in China. These dance therapists together with many other counsellors - are offering counselling services to clients. In China there are approximately one million counsellors trained within the national counselling training program but less than $5 \%$ of these are really active with a practice. Dance therapists offer their services to schools, hospitals, community centres, rehabilitation centres, senior's service centres and corporate organizations, etc. As the body/mind concept is rooted deeply within Chinese culture, people are often quite open and so resonate well to dance therapy as an 'imported' element from the West; this makes it easier for dance therapists to practice in China. Unfortunately, since there is no nationally-recognized credential or standard of training, quality control for the practice of this artistic discipline is still of concern. In Taiwan and Hong Kong, dance therapists hold licensed qualifications in psychological counselling and social work since their dance therapy credentials are not fully recognized by local authorities.

Currently, DMT research in China remains at a preliminary stage. Most DMT practitioners are not aware of the importance of research in this area and there's also a marked lack of trained researchers in this field. Inspirees Institute has therefore become a leading advocate for DMT research and has initiated several arts - and evidence-based 
research projects with its educational and medical partners - institutions which are working both nationally and internationally on the application of dance therapy for a range of different conditions including Parkinson's, schizophrenia and autism etc. Inspirees Institute has also established an exchange program which sends Chinese students and researchers abroad in order to further their research and training in this area. Another milestone is the establishment of the international peer reviewed journal CAET (Creative Arts Education and Therapy - Eastern and Western Perspectives) which bridges China and abroad and facilitates collaborative international research in this field. In Taiwan, Prof. Lee specializes in body image research. The Creative Body Image Program has been sponsored by a Ministry of Science and Technology Grant from 2013 to 2016 and is an evidence-based program with a wide range of benefits for young adult women and female adolescents - benefits including improvements in body confidence, self-expression, self-esteem, body awareness and creativity. In 2018, the Ministry of Science and Technology provided further grant aid in order for the project to establish a psychometric assessment tool named the "Dongshi body Image Scale". In Hong Kong, Professor Rainbow Ho is the leading researcher in this field and has produced more than 200 publications - including a recently controlled study on the efficacy of dance therapy on patients with cancer and dementia.

In China, dance therapy is developing quickly due to the demands of a quickly changing and expanding society - one which offers excellent opportunities for dance therapists to practice and experiment in various fields. There is great potential for this profession to develop in the future. However, dance therapy, together with other creative arts therapies, is still in its very early stages of development and is not yet fully recognized as an artistic discipline. The formation of a professional DMT association - together with the standardization of its training and qualifications - needs to be firmly established in order to consolidate its remarkable resources and develop a sense of creative synergy that will move it forwards. Thanks to the Chinese Groups of Arts Therapy established under the Chinese Psychological Society, DMT is moving gradually into the official mainstream. There are still very few male dance therapists - something which influences the establishment of a professional mainstream identity - and the fact that there is only one Masters-level non-academic training program limits the number of professionally trained dance therapists working in the country. There is, however, increasing interest from the university and it seems that it is now just a matter of time before an academic DMT program is in place.

\section{India}

In India, Tripura Kashyap pioneered dance movement therapy in India. After completing her course in 1980's she returned to India to work with varied populations in mental health settings. In the East of India, Sohini Chakraborty started dance therapy in the late 90's with women who were being sexually exploited. She created an organization called Kolkata Sanved in the year 2005, which works primarily for women \& children at-risk to trafficking, children of violence, mental health areas and children in the judicial system. As internationally trained therapists returned to India, they spread out across the country. 
In the north of India, Rashi Bijlani, Ritushree and Anshuma Kshetrapal started establishing DMP as a field, while in the west; Devika Mehta and Dilshad Patel are making their mark. Preetha Ramasubramanian and Tarana Khatri work in South India towards the development and acceptance of dance movement therapy within mental health spaces.

India does not have a professional master's level training program. There are two Diploma level programs offered currently for the duration of one year. One by Creative Movement Therapy Association of India in association with Meera College (affiliated to Savitribai Phule University, Pune) and Artsphere. Most of the internationally certified pioneers teach on and have been a part of the CMTAI course. The other program is offered by Kolkata Sanved in association with Tata Institute of Social Sciences, Mumbai and Kolkata. There are several unaccredited certificate and foundation level courses that are run by private organizations across the country. There is a slight distinction in the professional titles based on their level of training, which due to the lack of any regulatory body is not followed by many. Those with basic certificate training are DMT facilitators or creative movement therapy facilitators as compared to those who have a master's level training from USA or UK who call themselves dance movement therapists and dance movement psychotherapists respectively.

For dance movement therapy, there is no official government recognized professional body that can accredit and regulate dance movement therapy courses and professionals. Creative Movement Therapy Association of India, is a not- for profit organization that organizes courses and annual international conferences in India. It was founded in 2014. They have a founding team, an executive team and an advisory board to grow and develop professionals the field of creative/dance movement therapy in India. This though is not a professional body that provides licensure like the Association of Dance Movement Psychotherapy, UK. The practice of supervision, personal therapy and continuous professional development is mostly non-existent amongst facilitators. They are undertaken by those maintaining licensure from other international bodies of dance therapy.

In India, Tripura Kashyap worked in multiple mental health setups and schools across the country. Sohini Chakraborty since the 90's has been working with women exploited in trafficking. Devika Mehta works in the development of neuro-rehabilitation programs and neuro diversity programs across the country. Dilshad Patel has developed programs combining elements of DMT with sports medicine. Tanvi Bajaj is using LMA in developing work in the sectors of education and corporate trainings. Brinda Jacob Janvrin is a movement based expressive arts therapist and is known in the country for her work with Authentic movement.

India has an online magazine, https://cmtaisite.wordpress.com and private organizations are conducting researches at their organizational level. These however have not made it to any publication recognized by universities or professional bodies. Most of the current research being done is in the realm of therapeutic dance and application of dance forms as therapy rather than dance movement therapy. As all the practitioners working in India are still fairly young, there is a strong need for collaboration in order to produce, document and publish quality research papers of their work in this field.

Currently in India, the only body certifying mental health practitioners is RCI (Rehabilitation Council of India). Due to the demographic and geographic diversity in 
India, it is challenging for RCI and the government to come up with policies to regulate the mental health profession. It is because of lack of licensing that so many courses are being carried forth without common consensus on training material, teachers' level of training and training modules. This may lead to poor quality of professionals being trained in the country and minimal to no supervision and regulation of their practice. Some of the biggest challenges that we face are lack of infrastructure, funding as well as poor research in the field. Like many other countries, DMPs in India have to work in multiple set-ups to sustain themselves financially. Due to lack of awareness of effectiveness of DMP, they have to build programs from ground zero to be able to create jobs in this field.

\section{Japan}

In Japan, DMT consciously began in the 1970s. Psychiatric patients underwent DMT treatment mainly in hospitals including Saitama, Tokyo, Iwate, and Aomori. The original Japanese DMT took place without knowledge of American and European DMT theories and practices. This group discontinued their practices when the Japan Dance Therapy Association (JADTA) was established in 1992.

Japan does not have an official educational course of dance therapy at the graduate level. Therefore, there are several courses available to earn credits to become a dance therapist. JADTA organizes training course at regular level.

In 1992, JADTA was established by those who began to practice DMT in 1980s and are now recognized as leaders in this field. Though DMT gradually became popular, dance therapists faced difficulties obtaining employment in Japan, even when individuals obtained qualifications in dance therapy in the USA or Europe. Since 1999, the JADTA established three levels of qualification for members who fulfil standard requirements. There are 301 members (63 male, 238 female) as of February 17th 2017. Among them, there are 32 registered dance therapists, 5 registered associate dance therapists and 308 dance therapy facilitators.

In 1980s, DMT grew and was simultaneously conducted throughout Japan, especially in Tokyo, Shiga, Kyoto, Nara, and Osaka. However, as there was no networking among practitioners, each carried out his or her activities by themselves. The focus of DMT started to shift from psychiatric patients to students, patients with psychosomatic complaints, the elderly, and the public. With the establishment of JADTA in 1992, the profession started to expand nationally and later increased its international exposure and communication from 2010s.

\section{South Korea}

In South Korea, in 1990s Dr. Boon Soon Ryu, ADTR (BC-DMT) established Korea Dance/Movement Psychotherapy Association. It was the first move to introduce western DMT in Korea. In 2001, Seoul women's University opened Master program major in Dance/Movement Therapy. It was first set up in academic program. Later on, Hee Kyung Nam, Heeah Choi, Kyung Soon Ko, Nayung Kim, and Sang Myung Lee who had studied 
and received DTR and ADTR(R-DMT and BC-DMT) in late 1990s and early 2000s in US got back to Korea established Korean Society of dance/movement Psychotherapy in 2010.

South Korea has five university programs in master degree, majoring in Dance therapy. Especially Seoul women's university has master and Ph.D. program in DMT major has been established since 2001. Besides, there is ADTAAlternate Route training program initiated by Park Sunyoung. DMT professional associations offer their own DMT courses to register for Dance therapist certification of their own association. In South Korea there is not national qualification system in Arts therapy field as well as psychotherapy field. However all private certifications must deliberate and register to Korea Research Institute for Vocational Education and Training by law.

In South Korea, from 1990s to now several DMT professional associations established, their members has been growing and their certified dance therapist have been employed variety field. In South Korea, DMT has been conducted under supervising of medical profession in the hospital like China. Therefore it has not grown much, even though it is still conduced in mental hospital. DMT has been shifted to variety setting: school setting for maladjustment, nursing home for elderly, self-care and self-growth for public. Furthermore, after Sewol ferry sank, a huge tragic event, South Korean really cares about our trauma. Many trauma therapies are focusing on body-mind connection so that DMT expands as trauma interventions. Also, Korea Arts and Culture Education Service has offered arts healing programs and projects for several population. It is great opportunity as a DMT profession

Many DMT researches are conducted in master thesis in South Korea. Not only major in DMT, but also major in dance education, nursing, counseling and physical education. Some of DMT associations publish their own Journals, but it is of small scale and irregular publication yet.

In South Korea, DMT profession has been growing and recognizing in public. However, compare to Art and Music therapy, DMT is not enough yet. Art and Music therapy professions are interested in body and integrating body for their intervention. Recently there are some attempts to integrate between Dance, Art, and Music in therapy. It would be great new creativity for our professions and public.

\section{Vietnam}

\section{A culture of dance}

The desire for peace as well as the need to communicate and connect to self, community, and universe through creativity was depicted on Đông Sơn bronze drum at Van Lang the oldest kingdom of Vietnam - a millennia ago. A circle of flying bird figures, the shining sun and special ritual dances on the drum are poetic reminders of what it means to be human.

The concept of dance and movement as healing is woven into Vietnamese culture and the lives of the people. At the dawn of Vietnamese civilization, spiritual life, dancing, music and medicine were in unison. Vietnam today still maintains the roots of this 
unison, appearing in worship of the Mother Goddess (Đạo Mẫu)-a Vietnamese folk religion. The Mother Goddess ritual dance (Lên Đồng) harmonizes purity, joy and beauty - expressing the stories of deities or national heroes in their incarnations through dance/movement, songs and live music. In this tradition, dance is a form of medicine in which delight and the release of sorrow are able to transform physical, emotional and mental suffering into peace.

Moreover, dance is a part of life in Vietnam. At festivals, we dance and sing to celebrate special events in life or to give thanks to the Divine for the rain, sun and harvest. Dance is the means to dialogue and connection with our authentic self and nature.

The Vietnamese master of mindfulness, the monk Thích Nhất Hạnh said "Happiness is the unison of thoughts, words and actions". The body is the temple of the spirit, and our authentic self. Mindful breathing is a daily life practice of Vietnamese people for a healthy and balanced life.

\section{Dance Movement Therapy springs in Vietnam}

Grounded in a culture of dance, Minh Bùi has a natural desire to move. As an independent educator - especially an art-based facilitator - she has observed that the educational structure in Vietnam often does not support students to have equal dialogue or to think critically in order to make their own decisions. She has searched for expressive movement and other creative art forms as channels to empower students to be free to express themselves as individuals and to develop inner leadership. She has wondered about education through the prism of human rights and personal development besides being prepared for the commercial market.

Since 2010, she has created innovative projects and led Vietnam's first-ever movement-based workshops for self-discovery in Hanoi. Her dance journey was kindled by her reading of a borrowed book, Dance as a Healing Art by Anna Halprin in 2011. Minh was grateful for the creative expressions and wisdom which empowered her (an educator without a professional dance background) to cross the ocean to pursue a Master's Degree in Dance/Movement Therapy (DMT) at Sarah Lawrence College in New York under the Fulbright Program 2013-2015.

Thousands of years ago, Vietnamese culture understood and valued what was therapeutic in dance and movement. Now is the time for the modern version of Dance/ Movement therapy from the United States to be introduced into Vietnam. It rekindles in people that deep inner knowing which has been forgotten by many in this hectic, stressful, and industrial life.

It was recently reported that some $15 \%-20 \%$ of Vietnam's population have mental disorders. However both doctors and psychotherapists feel that - if a large-scale survey were to be conducted - this figure would actually be much higher. Moreover, the number of Vietnamese people who have physical disabilities (as victims of Agent Orange, domestic violence and survivors of human trafficking) is increasing. These populations are primarily treated with medication or undergo self-treatment at home but most do not have any professional long-term mental health plan. 
There is a great need for mental health services yet no solid organization and licensing requirements exist. There is more "space" to move but no "boundary" to cross. Anyone can work as a "therapist" in Vietnam. This creates a great deal of confusion within society. On the other hand, a culture of "saving face" forces people to repress themselves (and their mental health issues) in order to keep their "reputation" and avoid social discrimination. The strong community-oriented values

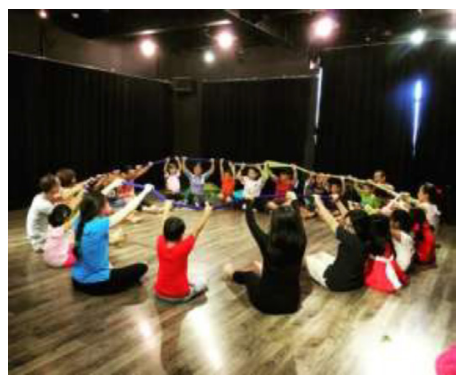
of Vietnam deepen the attitude that people have toward psychotherapy. Therapy is either something to be kept a secret (stigma) or avoided or the alternative belief is that anyone can give "advice".

Dance/Movement Therapy is an old yet new concept in Vietnam. Though people eagerly welcome and appreciate it, DMT has not yet found enough collective interest to be grounded in society. With no existing companion supervisors, classmates, or professors in this field in Vietnam, Minh is alone as she travels to seed the new ideas of DMT across diverse populations. As soon as she returned to her homeland in July, 2015, Minh began reaching out to H'mong children in the mountainous areas, to children with autism and special needs, to the LGBT community, to youth, and to professionals and psychotherapists as well as some NGOs.

Vietnamese Dance Movement Therapy (VDMT) group now has three active members: an arts-based educator with a background in psychology, a psychotherapist and Minh-R-DMT. About 100 innovative DMT workshops and projects utilizing body-movement for healing, empowering self-expression and inner leadership with personal, family, and groups have been organized. In 2018, the six-month project "Dance/Movement Therapy Approach to Children with Special Needs" will take place thanks to a small Fulbright Alumni grant from the US mission in Vietnam. From 23 to $30^{\text {th }}$ of September, we will host the first 3-day intensive DMT professional development training course; additionally, some introductory DMT workshops will be made available to members of the public. These will all be conducted by Kim Dunphy and Minh and the program will be supported by University of Melbourne, Australia.

VDMT is still in its infancy in Vietnam. We spontaneously interact with the needs of local people and society here. We have a goal for VDMT which is to be recognized and officially become part of the Vietnam Mental Health Services. We expect to enrich the lives of more and more people and to correspondingly enrich DMT with Vietnam's traditional dances and culture. We are also looking forward for more projects for our Train the Trainer courses and DMT programs to integrate DMT in education for both intervention and personal development.

\section{About the Authors}

Rashi Bijlani, MA, R-DMP (UK), Asha Hai \& Centre for Dance Movement Psychotherapy, India; Rainbow Tin Hung Ho, Professor, PhD, REAT, BC-DMT, CMA, University of Hong Kong; Devika Mehta, MSc, R-DMP (UK), M.A , St. Xavier's 
College, Mumbai and Synchrony, India. Nayung Kim, PhD, BC-DMT, CMA, Seoul Women's University, South Korea; Tsungchin Lee, Professor, National Chengchi University, Taiwan; Shoichi Machida, BC-DMT, Japanese Dance Therapy Association; Yukari Sakiyama PhD, BC-DMT, KMP Certified Analyst, Mukogawa Women's University, Japan. Tony Yu Zhou, PhD, CMA, Inspirees Institute, China/Netherlands; Pei-Shan Tsai, BC-DMT; GLCMA, NCC, Taiwan Dance Therapy Association.

\section{References}

Shoichi Machida, Yukari Sakiyama. (2017) Brief History and Current Status of Dance Movement Therapy in Japan. Creative Arts Education and Therapy, Vol (3)1, 64-68.

Machida, S. (2012) Dance Therapy no Gaiyou, Dance Therapy no Riron to Jissen, Karada to Kokoro eno Healing Art, Tokyo: the earth kyoikushinsya, 11-29.

Korean Dance Movement Psychotherapy Association. <http://www.kdmpa.com/bbs/board.php?bo_ table=history, 2018. 7.25>.

Korean Society of Dance/Movement Psychotherapy. <http://www.ksdmp.org/introduce/history.php, 2018. 7.25.>.

Nayung Kim. (2014) Dance Therapy, Korean Arts Professors Association, 2nd Workshop on Arts Therapy proceeding. 7-94.

Needham, J. (1964). Science and China's influence on the World. In R. Dawson (Ed.) The legacy of China. XIX. Oxford: Clarendon Press.

Northrop, F.S.C. (1946). The Meeting of East and West: an inquiry concerning world understanding (pp. 322-346). New York: The MacMillan Company

Russell, B. (1922). Chinese and Western civilization contrasted. In B. Russel The problem of China. XI, London: George Allen \& Unwin Ltd.

Schipper, K. (1994). The Taoist Body. Oakland: University of California Press

Tony Yu Zhou. (2015) The Circle of Learning: When West Meets East. Conference proceedings of ADTA Annual Conference, San Diego.

Lee, T.C. (2014) The Development of Dance Therapy in Taiwan: A Review and Prospect. Taiwan Counseling Quarterly, Vol 6(4), 9-16.

Wells, H.G. (1971). The Outline of History: Being a Plain History of Life and Mankind. New York: Doubleday \& Company Inc. 